\title{
2D PHOTONIC CRYSTAL BASED BIOSENSOR USING RHOMBIC RING RESONATOR FOR GLUCOSE MONITORING
}

\author{
T. Suganya and S. Robinson \\ Department of Electronics and Communication Engineering, Mount Zion College of Engineering and Technology, India
}

\begin{abstract}
A $2 D$ photonic crystal based biosensor using rhombic ring resonator is proposed and designed for monitoring the concentrations of glucose. The optical characteristics of rhombic ring resonator is analyzed and predicted by Finite Difference Time Domain method. A new structure of rhombic ring resonator is formed by utilizing bi-periodicity in the design of $2 D$ silicon PC slab in hexagonal lattice with the size of $10 \mu \mathrm{m}$ and $12 \mu \mathrm{m}$ in $X$ and $Z$ direction, respectively. The sensing parameters are observed in the rhombic ring resonator which are completely depends on the refractive indices of the material used. Thereby the concentrations of glucose can be monitored. The determined sensing parameters such as resonant wavelength is about $1546 \mathrm{~nm}$, quality factor is of 178.5, higher sensitivity of 1000nm/RIU and 100\% of transmission efficiency is also achieved.
\end{abstract}

\section{Keywords:}

Photonic Crystal, Biosensor, Rhombic Ring Resonator, Sensitivity

\section{INTRODUCTION}

Generally, photonic crystals (PC) play a significant role for device miniaturization. PCs are periodic artificial materials, which cannot available naturally. The relative permittivity of the material is varied periodically with low and high dielectric value [1]. Photons propagate through structure or sometimes they do not propagate which depends on wavelength. The periodic structure do not allow certain range of wavelength which is termed as Photonic Band Gap (PBG). The propagation of electromagnetic waves inside the PBG region is zero. The photonic bandgap depends on three factors such as dielectric constant $(\Delta)$, lattice constant $(a)$ and radius of the rod $(r)$. By these factors waves are allowed to pass through the structure at particular wavelength or frequency. The two types of lattices in photonic crystal are cubic and hexagonal lattice. The photonic crystal is of three types, based on the geometry of structure such as 1D, 2D and 3D. Among these 2D photonic crystals are highly efficient as it provides an accurate bandgap calculation, better light confinement etc.

Biosensors are the devices which can be used to detect and analyze the samples with different characteristics [2]. They are more attractive due to its features like sensitivity, speed, accuracy etc. In optical sensing two approaches have been utilized namely, the impact of resonant wavelength shift scheme and intensity variation scheme. The shift of the resonant wavelength leads to higher sensitivity. Also based on the way in which samples to be tested, sensing mechanism is of two, such as homogeneous and heterogeneous or surface sensing. The heterogeneous sensing mechanism is employed here for monitoring the glucose samples.

The applications of photonic crystal based sensors are as follows, chemical sensing, force and strain sensing [3], refractive index and gas sensing [4], dengue virus detection [5], pressure sensing [6], aqueous environment [7] and biosensing (protein, avidins, BSA, DNA, blood constituents, glucose monitoring, etc.)
[8]. Photonic crystal based devices can also be designed for several applications such as filters [9], demultiplexers [10], switches [11], optical logic gates [12], polarization converters etc. [13].

In 2010, Hsiao and Lee have designed photonic crystal with nano ring resonator using hexagonal lattice of silicon rods for biochemical sensing where the biomolecules were trapped and the cumulative refractive index is varied. Then, the output resonant wavelength is shifted based on their refractive index change. In this they have achieved the quality factor as 3000 whereas sensitivity is about only $6 \mathrm{~nm} / \mathrm{RIU}$ [14]. In 2011, Pal et al. have designed PC with nano cavity coupled waveguides where the biosensing possibility is investigated by detecting human IgG molecules to achieve better sensitivity is of $64.5 \mathrm{~nm} / \mathrm{RIU}$ [15]. Olyaee et al. have proposed photonic crystal with nano cavity of hexagonal lattice using silicon rods. The biomaterials which are suspended in a liquid medium inside nano-cavities tends to effective refractive index changes which lead to the resonant wavelength shift in the output terminal. Here the quality factor is about $4793.6 \mathrm{~nm} / \mathrm{RIU}$, then transmission efficiency is about only $75 \%$ and the sensitivity is about $65.7 \mathrm{~nm} / \mathrm{RIU}$ [16].

Dorfner et al. have also designed the photonic crystal with nano-cavity to analyze the concentrations of BSA. Drop filters have been added to improve sensitivity as $103 \pm 1 \mathrm{~nm} / \mathrm{RIU}$ [17] which is better than the sensitivity of $65.7 \mathrm{~nm} / \mathrm{RIU}$. Further Kim et al. have reported the design of surface emitting photonic crystal with point band edge laser to investigate the performances of different refractive indices of liquids from 1.296-1.372 with span of 0.019 . Thereby the sensitivity is getting improved as 135nm/RIU [18]. Moreover, in 2014, Najafgholinezhad and Olyaee have done an investigation on photonic crystal with microcavity resonator for analysis over the temperature dependency of water. There the achieved quality factor is of 15,000 but still sensitivity is of $141.67 \mathrm{~nm} / \mathrm{RIU}$ [19]. Thereby one thing can be noticed that sensitivity is inversely correlates with quality factor. Further, Dundar et al. have studied the concentrations of sugar water solution with nano cavity of hexagonal lattice. The achieved sensing parameters are as follows resonant wavelength is about $1475-1565 \mathrm{~nm}$, transmission efficiency is about $100 \%$ and sensitivity is of $280 \mathrm{~nm} / \mathrm{RIU}$ [20] better than the above.

Then, Kita et al. have demonstrated with nano lasers in hexagonal lattice for analyzing the performances of liquids with varying refractive indices from 1.00-1.37. The sensor is soaked into the liquids to obtain the sensitivity is about $350 \mathrm{~nm} / \mathrm{RIU}$ [21] and also resonant wavelength is in the range of $1570-1595 \mathrm{~nm}$. Here large amount of samples have needed to analyze the sensing parameters. Further, in 2016 to overcome above all criteria, M. S. Mohammed et al. have analyzed the PC with point defect in hexagonal lattice of $\mathrm{GaN}$ material to monitor the glucose concentrations. Here the resonant wavelength is about 1380$1440 \mathrm{~nm}$, average of quality factor is of $549.2,60 \%$ of transmission 
efficiency and over all higher sensitivity of $422 \mathrm{~nm} / \mathrm{RIU}$ is achieved in this literature [22].

Hence obviously, ultimate aim is to overcome and achieve higher order of sensing parameters. A 2D photonic crystal based biosensor is designed using rhombic ring resonator in hexagonal lattice for monitoring the glucose. The enhanced optical property material silicon is used here with the background of air which is better than GaN. The simulation result is obtained by Finite Difference Time Domain method [23]. Also higher order of sensing parameters can be achieved by this rhombic ring resonator such as resonant wavelength is in the range of $1540-1560 \mathrm{~nm}$, quality factor is of $178.6,100 \%$ of transmission efficiency and sensitivity is of $1000 \mathrm{~nm} / \mathrm{RIU}$. Hence the higher orders of sensing parameters are achieved in this paper which is greater than literatures reported before [14, 22, 15-21]. The rest of the paper is organized as follows, the proposed design is explained in section 2 , simulation results and discussions are reported in section 3 and section 4 concludes the paper.

\section{NUMERICAL ANALYSIS}

The photonic crystals exhibited property called as Photonic Band Gap. The photonic band structure can be termed as an optical insulator. Since the transmission of electromagnetic waves in certain range of frequencies are prohibited, i.e., there is no propagation of light over some order of wavelengths. The light propagation can be manipulated by the defect mechanism. The line defect in the ring resonator acts as waveguide. Thereby, the sensitivity can be enhanced. In the ring resonator structure, the resonance and the output power can be altered as the refractive index of the sample gets varied. The photonic crystal functions as sensor can be proved by solving Maxwell's electromagnetic equation [24].

$$
\nabla \times\left(\frac{1}{\epsilon} \nabla \times H\right)=\left(\frac{\omega}{C}\right)^{2} H
$$

In Eq.(1), $H$ is the magnetic field and $C$ is the speed of light. Also, $\varepsilon$ is the permittivity (dielectric function $\varepsilon=\eta^{2}$ or $\eta=\sqrt{\varepsilon}$ where $\eta$ is the refractive index), $\omega$ is the frequency of resonance. It is observed that when the dielectric function changes the frequency also changes. From the Eq.(1) it is noticed that dielectric function $\varepsilon$ is inversely proportional to frequency $\omega$. The sensing performances of the structure can be analyzed by the Eq.(2) [25]:

$$
L_{\text {eff }}=Q \lambda /(2 \pi \eta)
$$

In Eq.(2), $L_{\text {eff }}$ is the effective interaction length, $Q$ is the resonator quality factor, $\lambda$ is the resonant wavelength, $\eta$ is the refractive index of ring resonator.

\section{PROPOSED DESIGN}

\subsection{PHOTONIC BAND GAP}

The Fig.1 depicts the band diagram of hexagonal lattice without introducing any defects. The defects are nothing but breaking the periodicity by removing a row of rods (line defects) or single rod (point defect), changing the shape and size of the rods present in the structure and altering the structural parameters.
The band diagram has a PBG of TE mode and TM mode at different wavelength ranges which is listed in the Table.1. We have considered the wavelength range from $1125 \mathrm{~nm}$ to $1725 \mathrm{~nm}$ as it belongs to third window since it is highly efficient in the communication systems. The first TE PBG is accounted for further investigation, which is clearly pictured in blue colored region.

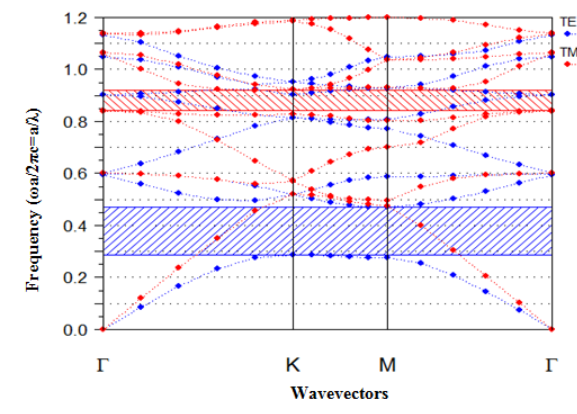

Fig.1. Band Diagram of Proposed Device

Table.1. Frequency and Wavelength from PBG

\begin{tabular}{|c|c|c|}
\hline Types of PBG & Frequency $(\mathbf{a} / \boldsymbol{\lambda})$ & Wavelength $(\mathbf{n m})$ \\
\hline TE & $0.480-0.313$ & $1125-1725 \mathrm{~nm}$ \\
\hline TM & $0.954-0.865$ & $566-624 \mathrm{~nm}$ \\
\hline
\end{tabular}

The horizontal and vertical axes in the band diagram represent the wavevector and normalized frequency, respectively. The wavevector is calculated in the Brillouin zone, which is equal to the entire periodic structure. The normalized frequency of the PC structure is $\omega a / 2 \pi c=a / \lambda$, where, $\omega$ is the angular frequency, $a$ is the lattice constant, $c$ is the velocity of light in free space and $\lambda$ is the free space wavelength.

\subsection{PC BASED BIOSENSOR}

The Fig.2 depicts the designed photonic crystal based biosensor using rhombic ring resonator which consists of the hexagonal array of circular silicon rods placed in a background of air. In the hexagonal lattice, the total number of rods in $\mathrm{X}$ and $\mathrm{Z}$ direction are 21 and 21 , respectively.

The proposed sensor consists of two optical waveguides namely bus waveguide and dropping waveguide. The bus waveguide acts as an input port placed at one end whereas dropping waveguide acts as an output port at another end. Both the waveguides can be designed by utilizing bi-periodicity.

Bi-periodicity is nothing but reducing the radii of rods in specific manner, such rods having radii of 50nm. Rhombic ring resonator is the heart of the proposed biosensor which is placed at the centre. It consists of two rings such as inner ring and outer ring rods. Both the radii of inner ring and outer ring rods are $105 \mathrm{~nm}$. Centre elliptical rod is of $150 \mathrm{~nm}$. The distance between the two rod is about $547 \mathrm{~nm}$, it is also called as lattice constant denoted by $a$ and the dielectric constant of Si rod is 11.97 (refractive index = 3.46).

The Fig. 3 shows 3D view of proposed sensor. The size of sensor is about $11.4 \mu \mathrm{m} \times 9.8 \mu \mathrm{m}$. The simulation parameters of the sensor are listed in Table.2. 


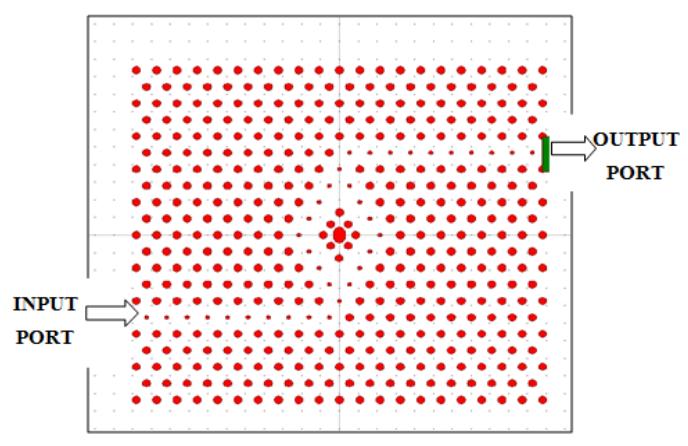

Fig.2. Schematic Representation of Proposed Biosensor

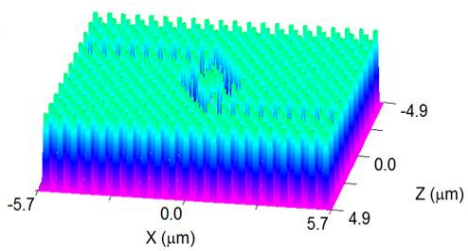

Fig.3. 3D View of Proposed Biosensor

Table.2. Simulation Parameters of Sensors

\begin{tabular}{|c|c|}
\hline Design Parameters & Values \\
\hline Configuration & Rod in air \\
\hline Rod shape & Circular \\
\hline Lattice structure & Hexagonal \\
\hline Lattice constant & $0.54 \mu \mathrm{m}$ \\
\hline Radius of rod & $0.1 \mu \mathrm{m}$ \\
\hline Refractive index of rod & 3.46 \\
\hline Dielectric constant of Si rod & 11.97 \\
\hline
\end{tabular}

When the light is propagating from bus waveguide (input port), through the rhombic ring resonator of sensor which is filled the sample, there is some molecular interactions due to the changes in the concentration of glucose sample results in variation of refractive indices of sample taken. Correspondingly, there is shift in the resonant wavelength along with the changes in the transmitted power. These can be observed at dropping waveguide (output port). Thereby better quality factor and sensitivity can be obtained. The sensitivity is defined as the resonance wavelength shift $\Delta \lambda$ per analyte refractive index change $\Delta n[22]$.

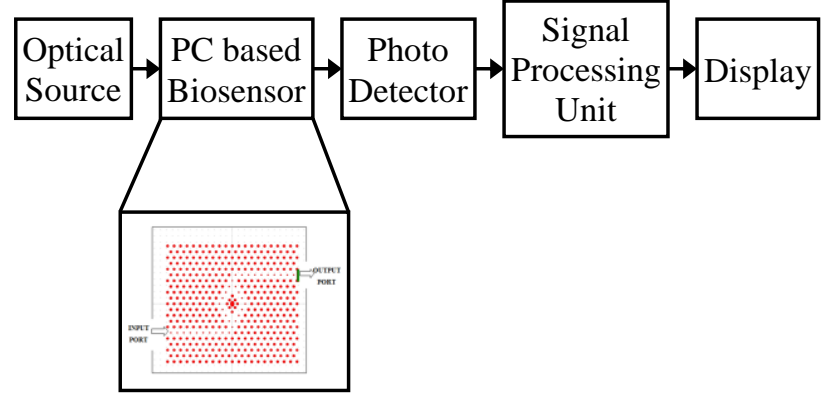

Fig.4. Schematic Structure of Glucose Sensor
The schematic representation of sensing mechanism is shown in Fig.4 consists of several components such as optical source, photonic crystal based biosensor, photo detector, signal processing unit and display. Optical source is the one by which the optical light is produced then reached in to the sensor where light is getting propagated through the glucose samples taken. Photo detector is used to convert electrical signal into optical signal. Then the output is processed in the signal processing unit. The processing includes filtering, modulation, demodulation, phase shifting, frequency conversion etc. Based on desired output the functions may be carried out in it. Finally output will be displayed in the display.

\section{SIMULATION RESULTS AND DISCUSSION}

When there is shift in refractive index of glucose sample then correspondingly there is shift in the resonance wavelength. According to that, there is shift in the level of glucose concentration. In such a way the sensor has to be designed. The normalized transmission spectrum of the sensor at 0 glucose level is shown in Fig.5. From that, obtained resonant wavelength, quality factor and transmitted efficiency is about $1545 \mathrm{~nm}, 171.6$ and $100 \%$, respectively. The light signal is launched into the input port. The output signal power reaches the power monitor which is positioned at the output port is normalized by the input signal power.

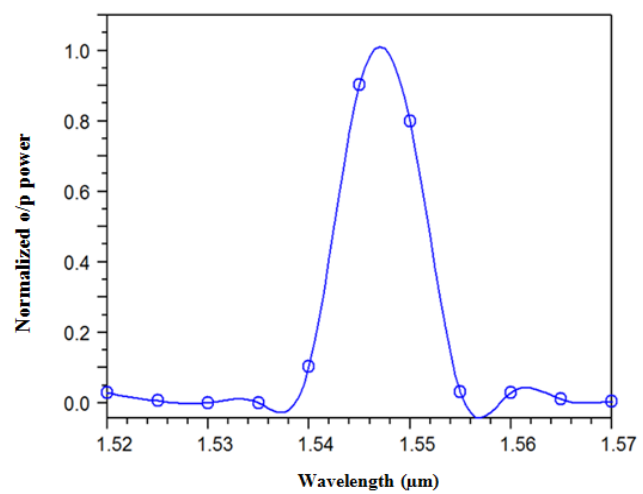

Fig.5. Normalized Transmission Spectrum of the Proposed Sensor at $0 \mathrm{~g} / \mathrm{L}$

The electric field distribution of the proposed sensor at ON resonance and OFF resonance is representing in Fig.6(a) and Fig.6(b), respectively. It is clear that the signal couples in to the sensor at the resonance wavelength. The signals getting couples only at and above the resonance wavelength. This condition can be termed as ON resonance. When the signal is not able to couple below the resonant wavelength is about at $1300 \mathrm{~nm}$ then that condition is called OFF resonance.

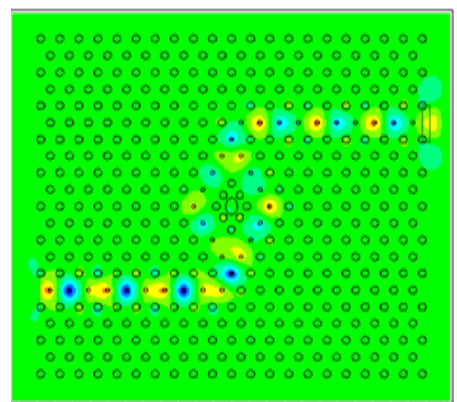

(a) $\lambda=1560 \mathrm{~nm}$ 


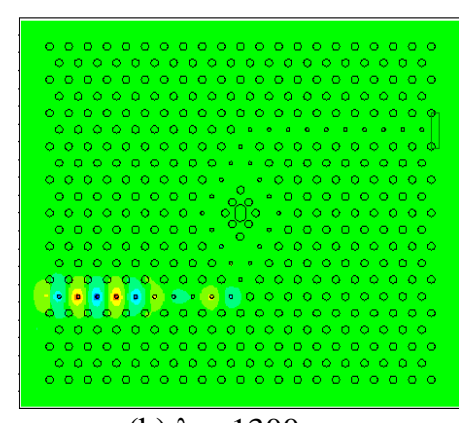

(b) $\lambda=1300 \mathrm{~nm}$

Fig.6. Schematic Representation of Field Distribution of sensor at (a) ON resonance (b) OFF resonance

The normalized output spectrum of proposed sensor is depicted in Fig.6. It is noticed that when the value of refractive index gets increase then resonance wavelengths also getting shifted accordingly.

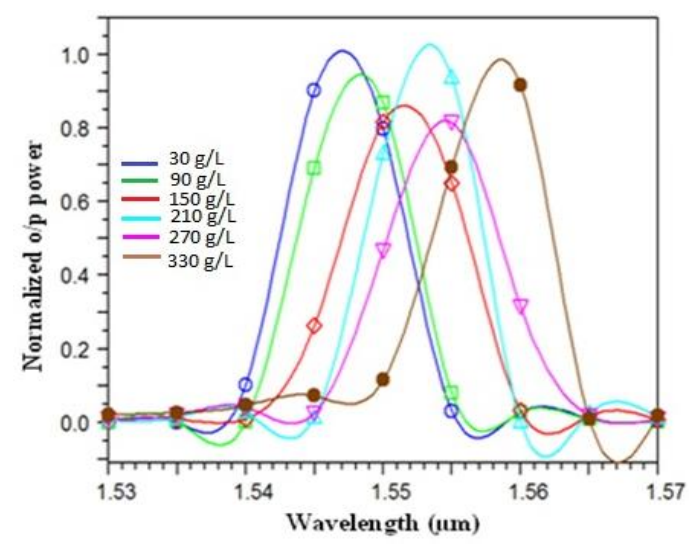

Fig.7. Normalized Transmission Spectrum of the Proposed Sensor

At $30 \mathrm{~g} / \mathrm{L}$ of glucose, the achieved resonant wavelength is about $1546 \mathrm{~nm}$, whereas other sensing parameters such as sensitivity is of $333.3 \mathrm{~nm} / \mathrm{RIU}$, quality factor is about 171.7 and transmitted efficiency is of $100 \%$. Also it is investigated that there is about $2.5 \mathrm{~nm}$ of resonant wavelength shift is noticed for every increasing of $30 \mathrm{~g} / \mathrm{L}$ of glucose concentration. The refractive index, resonant wavelength, sensitivity, quality factor and transmission efficiency of the proposed biosensor at different glucose concentration is listed in Table.3. The maximum sensitivity, quality factor and transmission efficiency are 1000nm/RIU, 178 and 100\%, respectively.

Table.3. Refractive Index, Resonant Wavelength, Sensitivity, Quality Factor and Transmission Efficiency of the Sensor at Different Glucose Concentration

\begin{tabular}{|c|c|c|c|c|c|}
\hline $\begin{array}{c}\text { Glucose } \\
\text { Concentration } \\
(\mathbf{g} / \mathbf{L})\end{array}$ & $\begin{array}{c}\text { Refractive } \\
\text { Index }\end{array}$ & $\begin{array}{c}\text { Resonant } \\
\text { Wavelength } \\
(\boldsymbol{\mu m})\end{array}$ & $\begin{array}{c}\text { Sensitivity } \\
(\mathbf{n m} / \mathbf{R I U})\end{array}$ & $\begin{array}{c}\text { Quality } \\
\text { Factor }\end{array}$ & $\begin{array}{c}\text { Transmission } \\
\text { Efficiency } \\
(\boldsymbol{\%})\end{array}$ \\
\hline 30 & 2.460 & 1.546 & 333.3 & 171.7 & 100 \\
\hline 90 & 2.466 & 1.548 & 666.6 & 110.5 & 80 \\
\hline 150 & 2.472 & 1.551 & 1000 & 155.1 & 80 \\
\hline 210 & 2.478 & 1.553 & 666.6 & 178.5 & 100 \\
\hline 270 & 2.484 & 1.555 & 666.6 & 153.9 & 80 \\
\hline 330 & 2.490 & 1.558 & 1000 & 173.1 & 95 \\
\hline
\end{tabular}

\section{CONCLUSION}

The two dimensional photonic crystal based biosensor is designed and its sensing characteristics are analyzed. The sensor is designed using two dimensional photonic crystals with the hexagonal lattice of circular silicon rods surrounded by air. The sensor is designed in the wavelength range $1540 \mathrm{~nm}$ to $1560 \mathrm{~nm}$. The resonance wavelength, sensitivity, Q factor and output power of the sensor are $1546 \mathrm{~nm}, 1000 \mathrm{~nm} / \mathrm{RIU}, 178.5$ and $100 \%$ respectively. The designed sensor is highly sensitive to refractive index. By knowing the refractive index value, the glucose concentration is obtained. The low level of urea excretion by kidney is indicator of kidney related diseases and the high level of urea excretion is due to higher protein intake and breakdown. So maintaining the glucose level in our body is really an important thing. Hence the sensor is designed in such a way to obtain the higher sensitive output.

\section{REFERENCES}

[1] John D Joannopoulous, "Photonic Crystals Molding the Flow of Light", $2^{\text {nd }}$ Edition, Princeton University of Press, 2008.

[2] Florinel-Gabriel Banica, "Chemical Sensors and Biosensors: Fundamental and Applications", WileyBlackwell, 2012

[3] T.T. Mai, F.L. Hsiao, C. Lee, W. Xiang, C. Chenc and W.K. Choi, "Optimization and Comparison of Photonic Crystal Resonators for Silicon Microcantilever Sensor", Sensors and Actuators, Vol. 165, No. 1, pp. 16-25, 2011.

[4] C. Kang, C. Phare and S.M. Weiss, "Photonic Crystal Defects with Increased Surface Area for Improved Refractive Index Sensing”, Proceedings of Conference on Laser and Electro Optics and Quantum Electronics and Laser Science, Vol. 16, No. 21, pp. 1-2, 2010.

[5] S. Mandal, J. Goddard and D. Erickson, "Nanoscale Optofluidic Sensor Arrays for Dengue Virus Detection", Proceedings of Conference on Laser and ElectroOptics/Conference on Quantum Electronics and Laser Science, Vol. 18, pp. 1-2, 2008.

[6] O. Saeed and D.A. Asghar, "High Resolution and Wide Dynamic Range Pressure Sensor based on TwoDimensional Photonic Crystal", Photonic Sensors, Vol. 2, No. 1, pp. 92-96, 2012.

[7] F. Abdel Malek, "Design of A Novel Left-Handed Photonic Crystal Sensor Operating in Aqueous Environment", IEEE Photonics Technology, Vol. 23, No. 3, pp. 188-190, 2011.

[8] C. Lee, A. Sueh, P. Yee, J.L. Perera, C. Chen and N. Balasubramanian, "Design of Nanobiophotonics Resonators for Biomolecules Detection", Proceedings of IEEE International Conference on Nano Micro Engineered and Molecular Systems, pp. 274-279, 2008.

[9] H. Alipour-Banaei and F. Mehdizadeh, "A Proposal for Anti-UVB Filter based on One Dimensional Photonic Crystal Structure", Journal of Nanomaterials and Biostructures, Vol. 20, No. 7, pp. 361-367, 2012.

[10] X. Zhang, Q. Liao, T. Yu, N. Liu and Y. Huang, "Novel Ultracompact Wavelength Division Demultiplexer based on Photonic Band Gap", Optics Communication, Vol. 285, No. 17, pp. 274-276, 2012. 
[11] Ahmed Sharkawy, Shouyuan Shi and Dennis W. Prather, "Electro Optical Switching using Coupled PC Waveguides", Optics Express, Vol. 10, No. 6, pp. 1048-1059, 2002.

[12] P. Andalib and N. Granpayeh, "All Optical Ultracompact Photonic Crystal and Gate based on Nonlinear Ring Resonator", Journal Optics Society, Vol. 26, No. 12, pp. 1016, 2009.

[13] M.F.O. Hameed, M. Abdelrazzak and S.S.A. Obayya, "Novel Design of Ultra-Compact Triangular Lattice Silica Photonic Crystal Polarization Converter", IEEE Journal Lightwave Technology, Vol. 31, No. 1, pp. 81-86, 2013.

[14] F. Hsiao and C. Lee, "Computational Study of Photonic Crystals Nano-Ring Resonator for Biochemical Sensing”, IEEE Sensors, Vol. 10, No. 7, pp. 1185-1191, 2010.

[15] Saeed Olyaee, Samira Najafgholinezhad and Hamed Alipour Banaei, "Four-Channel Label-Free Photonic Crystal Biosensor using Nano Cavity Resonators", Photonic Sensors, Vol. 3, No. 3, pp. 231-236, 2013.

[16] S. Pal, E. Guillermain, R. Sriram, B.L. Miller and P.M. Fauchet, "Silicon Photonic Crystal Nano Cavity Coupled Waveguides for Error-Corrected Optical Biosensing", Biosensors and Bioelectronics, Vol. 26, No. 19, pp. 40244031, 2011.

[17] D. Dorfner, T. Zabel, T. Hurlimann, N. Hauka, L.Frandsen, U. Rant, G. Abstreiter, and J. Finley, "Photonic Crystal Nanostructures for Optical Biosensing Applications", Biosensors and Bioelectronics, Vol. 24, No. 15, pp. 36883692, 2009.

[18] S. Kim, J. Lee, H. Jeon and H. J. Kim, "Fiber Coupled Surface-Emitting Photonic Crystal Band Edge Laser for
Biochemical Sensor Applications", Applied Physics Letters, Vol. 94, No. 133, pp. 240-247, 2009.

[19] S. Najafgholinezhad and S. Olyaee, "A Photonic Crystal Biosensor with Temperature Dependency Investigation of Micro-Cavity Resonator", Optik-International Journal for Light and Electron Optics, Vol. 125, No. 27, pp. 6562-6565, 2014.

[20] M.A. Dundar, E.C.I. Ryckebosch, R. Notzel, F.Karouta, L.J. Van Ijzendoorn and R.W. Vander Heijden, "Sensitivities of in GaAsP Photonic Crystal Membrane Nano Cavities to Hole Refractive Index", Optics Express, Vol. 18, No. 5, pp. 4049-4056, 2010.

[21] S. Kita, K. Nozaki and T. Baba, "Refractive Index Sensing Utilizing a CW Photonic Crystal Nano Laser and its Array Configuration", Optics Express, Vol. 16, No. 11, pp. 81748180, 2008.

[22] M S Mohamed, Mohamed Farahat O. Hameed, Nihal F.F. Areed, M.M. El-Okr and S.S.A. Obayya, "Analysis of Highly Sensitive Photonic Crystal Biosensor for Glucose Monitoring”, ACES Journal, Vol. 31, No. 7, pp. 25-27, 2016

[23] H. Butt, Q. Dai and T.D. Wilkinson, "Photonic Crystals and Meta Material Filters based on 2D Arrays of Silicon NanoPillars", Progress in Electromagnetics Research, Vol. 113, No. 42, pp. 179-194, 2011.

[24] V. Dinesh, "Analysis and Simulation of Photonic Crystal Components for Optical Communication", PhD Dissertation, 2003.

[25] Xudong Fan, Ian M. White, Siyka I. Shopova, Hongying Zhu, Jonathan D. Suter, Yuze Sun, "Sensitive Optical Biosensors for unlabeled targets: A Review", Analytica Chimica Acta, Vol. 620, No. 1-2, pp. 8-26, 2008. 